\title{
Analysis of a Single Species Model with Dissymmetric Bidirectional Impulsive Diffusion and Dispersal Delay
}

\author{
Haiyun Wan, Long Zhang, and Zhidong Teng \\ College of Mathematics and System Sciences, Xinjiang University, Urumqi 830046, China \\ Correspondence should be addressed to Long Zhang; longzhang_xj@sohu.com
}

Received 19 November 2013; Accepted 25 February 2014; Published 9 April 2014

Academic Editor: Yongkun Li

Copyright ( 2014 Haiyun Wan et al. This is an open access article distributed under the Creative Commons Attribution License, which permits unrestricted use, distribution, and reproduction in any medium, provided the original work is properly cited.

\begin{abstract}
In most models of population dynamics, diffusion between two patches is assumed to be either continuous or discrete, but in the real natural ecosystem, impulsive diffusion provides a more suitable manner to model the actual dispersal (or migration) behavior for many ecological species. In addition, the species not only requires some time to disperse or migrate among the patches but also has some possibility of loss during dispersal. In view of these facts, a single species model with dissymmetric bidirectional impulsive diffusion and dispersal delay is formulated. Criteria on the permanence and extinction of species are established. Furthermore, the realistic conditions for the existence, uniqueness, and the global stability of the positive periodic solution are obtained. Finally, numerical simulations and discussion are presented to illustrate our theoretical results.
\end{abstract}

\section{Introduction}

In the last few years, mathematicians and ecologists have been actively investigating the dispersal of populations, a ubiquitous phenomenon in population dynamics. Levin [1] showed that both spatial dispersal of populations and population dynamics are much affected by spatial heterogeneity. In real life, dispersal often occurs among patches in ecological environments; because of the ecological effects of human activities and industries, such as the location of manufacturing industries and the pollution of the atmosphere, soil, and rivers, reproduction- and population-based territories and other habitats have been broken into patches. Thus, realistic models should include dispersal processes that take into consideration the effects of spatial heterogeneity.

In recent years, increasing attention has been paid to the dynamics of a large number of mathematical models with diffusion, and many nice results have been obtained. The persistence and extinction for ordinary differential equation and delayed differential equation models were investigated in [2-6]. Global stability of equilibrium and periodic solution for diffusing model were studied in [7-12]. However, in all of above population dispersing systems, it is always assumed that the dispersal occurs at every time. For example, in [7],
Beretta and Takeuchi proposed the following single-species diffusion Volterra models with continuous time delays:

$$
\begin{aligned}
\dot{x}_{i}= & x_{i}\left(e_{i}-a_{i} x_{i}+\gamma_{i} \int_{-\infty}^{t} F_{i}(t-\tau) x_{i}(\tau) d \tau\right) \\
& +\sum_{\mu=1}^{n} D_{i \mu}(t)\left(x_{\mu}-x_{i}\right), \quad i \in N,
\end{aligned}
$$

where $N=\{1, \ldots, n\} n$ is the number of patches, and $x_{i}$ is the population density in the $i$ th patch. The form of the dispersal established in this model is continuous; that is, the dispersal is always happening at any time.

Actually, real dispersal behavior is very complicated and is always influenced by environmental change and human activities. In many practical situations, it is often the case that maybe one of the species suffers a significant loss or increase in density for some reason at some transitory time slots. These short-term perturbations are often assumed to be in the form of impulses in the modeling process. For example, when winter comes, birds will migrate between patches in search for a better environment, whereas they do not diffuse in other seasons, and the excursion of foliage seeds occurs during a fixed period of time every year. 
Therefore, impulsive differential equations [13] provide a natural description of such system. With the developments and applications of impulsive differential equations, theories of impulsive differential equations have been introduced into population dynamics, and many important studies have been performed [14-20].

In [14], the authors studied the following autonomous single-species model with impulsively bidirectional diffusion:

$$
\begin{aligned}
& \dot{x}_{1}(t)=x_{1}(t)\left[a_{1}-b_{1} x_{1}(t)\right], \\
& \dot{x}_{2}(t)=x_{2}(t)\left[a_{2}-b_{2} x_{2}(t)\right], \quad t \neq n \tau, \\
& \Delta x_{1}(t)=d_{1}\left(x_{2}(t)-x_{1}(t)\right), \\
& \Delta x_{2}(t)=d_{2}\left(x_{1}(t)-x_{2}(t)\right), \quad t=n \tau,
\end{aligned}
$$

where $a_{i}, b_{i}(i=1,2)$ are the intrinsic growth rate and density-dependent parameters of the population $x_{i}$ and $d_{i}$ is the dispersal rate in the $i$ th patch. Consider $\Delta x_{i}=$ $x_{i}\left(n \tau^{+}\right)-x_{i}\left(n \tau^{-}\right)$, where $x_{i}\left(n \tau^{+}\right)=\lim _{t \rightarrow n \tau^{+}} x_{i}(t)$ represents the density of population in the $i$ th patch immediately after the $n$th diffusion pulse at time $t=n \tau$, while $x_{i}\left(n \tau^{-}\right)=$ $\lim _{t \rightarrow n \tau^{-}} x_{i}(t)=x_{i}(\tau)$ represents the density of population in the $i$ th patch before the $n$th diffusion pulse at time $t=$ $n \tau$ ( $\tau$ the period of dispersal between any two pulse events is a positive constant, $n=1,2, \ldots)$. It is assumed here that the net exchange from the $j$ th patch to $i$ th patch is proportional to the difference $x_{j}-x_{i}$ of population densities. The dispersal behavior of populations between two patches occurs only at the impulsive instants $n \tau$. Obviously, in this model, species $x$ inhabits, respectively, two patches before the pulse appears, and when the time at the pulse comes, species $x$ in two patches disperses from one patch to the other. The boundedness and global stability of positive periodic solution were obtained.

Time delay often appears in many control systems (such as aircraft, chemical, or process control systems) either in the state, the control input, or the measurements. In order to reflect the dynamical behaviors of models that depend on the past history of system, it is often necessary to incorporate time delays into systems [21]. There have been extensive theoretical works on delay differential equations in the past three decades. The research topics include global asymptotic stability of equilibria, existence of periodic solutions, complicated behavior, and chaos (e.g., $[8,22-24])$.

Takeuchi et al. in [25] studied the following population model with time delays that introduced the dispersal time for individuals to move from one patch to other patches:

$$
\begin{array}{r}
\dot{x}_{i}(t)=x_{i}(t)\left[a_{i}-b_{i} x_{i}(t)\right] \\
+\sum_{j=1}^{n}\left[\varepsilon_{i j} d_{i j} x_{j}\left(t-\tau_{i j}(t)\right)-d_{j i}(t) x_{i}(t)\right], \\
i=1,2, \ldots, n,
\end{array}
$$

where $b_{i}, i=1, \ldots, n$, are positive constants, $d_{i j}, i, j=1, \ldots, n$, are nonnegative constants, $\varepsilon_{i j}=e^{\gamma_{i j} \tau_{i j}}$ satisfy $0<\varepsilon_{i j} \leq 1, a_{i}$, $i=1, \ldots, n$, are constants, and some of them may be negative.
In this paper, the authors took account of dispersal delay; however, they assumed that the dispersal is continuous.

It is well known that the application of impulsive delay differential equations to population dynamics has been an interesting topic since it is reasonable and correct in modelling the evolution of population, such as pest management [26].

However, in all of the impulsive dispersal models studied up till now, there are few papers considering the dispersal delay, which is really a pity. Actually, in the real world, the migration between patches is usually not immediate; that is, dispersal processes often involve time delay. For example, elks move from higher to lower elevations to escape cold in winter, and ungulates migrate annually among grazing areas to follow spatiotemporal changes in rainfall. Obviously, this kind of dispersal delay between patches extensively exists in the real world. Therefore, it is a very basilic problem to research this kind of population dynamic systems.

Moreover, in the above impulsive dispersal models, it is assumed that the dispersal occurs between homogeneous habitat patches; that is, the dispersal rate between any two patches is equal or symmetrical $[1,11]$, which is really too idealized for a real ecosystem. Actually, in the real world, due to the heterogeneity of the spatiotemporal distributions in nature, movement between fragments of patches is usually not the same rate in both directions. In addition, once the individuals leave their present habitat, they may not successfully reach a new one, due to predation, harvesting, or other reasons, so that there are traveling losses. Thus, the dispersal rates among these patches are not always the same. Rather, in real ecological situations they are different (or dissymmetrical) [27, 28].

Therefore, it is our basilic goal to investigate a single species model with dissymmetric impulse dispersal and dispersal delay. Motivated by the calculation hereinbefore, in this paper, we extend system (2) with dispersal delay and dispersal loss and consider it

$$
\begin{aligned}
& \frac{d x_{1}(t)}{d t}=x_{1}(t)\left[a_{1}-b_{1} x_{1}(t)\right], \\
& \frac{d x_{2}(t)}{d t}=x_{2}(t)\left[a_{2}-b_{2} x_{2}(t)\right], \quad t \neq n \tau, \\
& \Delta x_{1}(t)=d_{2} x_{2}\left(t-\tau_{0}\right)-D_{1} x_{1}(t), \\
& \Delta x_{2}(t)=d_{1} x_{1}\left(t-\tau_{0}\right)-D_{2} x_{2}(t), \quad t=n \tau,
\end{aligned}
$$

where $D_{i}(i=1,2)$ is the rate of population $x_{i}$ emigrating from the $i$ th patch and $d_{i}$ is the rate of population $x_{i}$ immigrating from the $i$ th patch. Here we assume $0 \leq d_{i} \leq$ $D_{i} \leq 1$, which means that there possibly exists mortality during migration between two patches. $\tau_{0} \leq \tau$ stands for the time delay; that is, a period of time of species $x$ dispersing between patches.

The organization of this paper is as follows. In Section 2, as preliminaries, the definition of permanence and some useful lemmas are introduced. From discrete dynamic system theory, we establish the stroboscopic map of system (4), by which we can obtain the dynamical behaviors of it. In 
Section 3, the results of permanence and extinction for the system are presented. The existence and the uniqueness of the positive periodic solution for system (4) are established in Section 4. In Section 5, using the discrete dynamic system theory in [29], we can get the global stability of the positive periodic solution for the system. Finally, we give a brief discussion and our theoretical results are conformed by numerical simulations.

\section{Preliminaries}

In this section we introduce a definition and some notations and state some results which will be useful in subsequent sections.

For any fixed $t \in R_{+}=[0, \infty)$, let $\phi(s):\left[t-\tau_{0}, t\right] \rightarrow R^{2}$ be a piecewise continuous function such that $\phi(s)$ is continuous in $s \neq n \tau, \phi\left(n \tau^{-}\right)=\lim _{s \rightarrow n \tau^{-}} \phi(s)=\phi(n \tau)$ and $\phi\left(n \tau^{+}\right)=$ $\lim _{s \rightarrow n \tau^{+}} \phi(s)$ exist. For any fixed $t \in R_{+}$, let $P C\left[t-\tau_{0}, t\right]$ denote the Banach space of all such piecewise continuous functions $\phi(\theta):\left[t-\tau_{0}, t\right] \rightarrow R^{2}$ with the norm $\|\phi\|=$ $\sup _{t-\tau_{0} \leq \theta \leq t}|\phi(\theta)|$. Further, let $P C_{+}\left[t-\tau_{0}, t\right]=\left\{\phi=\left(\phi_{1}, \phi_{2}\right) \in\right.$ $P C\left[t-\tau_{0}, t\right]: \phi_{i}(\theta) \geq 0$ for all $\theta \in\left[t-\tau_{0}, t\right]$ and $\phi_{i}(t)>0$ for $i=1,2\}$.

Motivated by the biological background of system (4), in this paper we always assume that all solutions of system (4) satisfy the following initial conditions:

$$
x_{i}(\theta)=\phi_{i}(\theta) \quad \forall \theta \in\left[-\tau_{0}, 0\right], i=1,2,
$$

where $\phi=\left(\phi_{1}, \phi_{2}\right) \in P C_{+}\left[-\tau_{0}, 0\right]$. For any $\phi \in P C_{+}\left[-\tau_{0}, 0\right]$, by the fundamental theory of impulsive functional differential equations $[30,31]$, system (4) has a unique solution $x(t, \phi)=\left(x_{1}(t, \phi), x_{2}(t, \phi)\right)$ satisfying the initial conditions (5).

Definition 1. System (4) is said to be permanent, if there are positive constants $m_{i}$ and $M_{i}$ such that

$$
m_{i} \leq \liminf _{t \rightarrow \infty} x_{i}(t) \leq \limsup _{t \rightarrow \infty} x_{i}(t) \leq M_{i}, \quad i=1,2,
$$

for any positive solution $x(t)=\left(x_{1}(t), x_{2}(t)\right)$ of system $(4)$.

We consider the following scalar impulsive differential equation:

$$
\begin{gathered}
\dot{x}(t)=f(t, x(t)), \quad t \neq n \tau, \\
x\left(n \tau^{+}\right)=I(x(n \tau)), \quad t=n \tau,
\end{gathered}
$$

where $t \in R_{+}, n \tau>0$, is an impulsive time sequence, $x \in R$, $f(t, x): R_{+} \times R \rightarrow R$ is continuous, and $I(x): R \rightarrow R$ is a nondecreasing function. We have the following comparison theorem $[30,31]$ for $(7)$.

Lemma 2. Let $x(t)$ be a solution of system (7) defined on $\left[t_{0}, T\right]$ and satisfy

$$
\begin{gathered}
\dot{u}(t) \leq(\geq) f(t, u(t)), \quad t \neq n \tau, \\
u\left(n \tau^{+}\right) \leq(\geq) I(u(n \tau)), \quad t=n \tau .
\end{gathered}
$$

If $u\left(t_{0}\right) \leq(\geq) x\left(t_{0}\right)$, then $u(t) \leq(\geq) x(t)$ for all $t \in\left[t_{0}, T\right]$.
Lemma 3 (see [32]). Consider the following nonlinear impulsive system:

$$
\begin{aligned}
\dot{x}(t) & =x(t)(a(t)-b(t) x(t)), \quad t \neq t_{k}, \\
x\left(t_{k}^{+}\right) & =\left(1-\theta_{k}\right) x\left(t_{k}\right), \quad t=t_{k}, k \in N, \\
x\left(0^{+}\right) & =x_{0},
\end{aligned}
$$

where $a(t)$ and $b(t)$ are bounded and continuous $\omega$-periodic functions defined on $R_{+}, b(t) \geq 0$ for all $t \in R_{+}$and impulsive coefficients $\theta_{k} \in(0,1), \theta_{k}=\theta_{k+q}, q$ is a fixed positive integer, and $t_{k+q}=t_{k}+\omega$. If $\int_{0}^{\omega} a(s) d s+\ln \prod_{i=1}^{q}\left(1-\theta_{i}\right)>0$, then there exists a unique positive periodic solution of system (9), which is globally asymptotically stable.

Remark 4. If system (9) degenerates into the following autonomous impulsive differential equation:

$$
\begin{aligned}
\dot{x}(t) & =x(t)(a-b x(t)), \quad t \neq t_{k}, \\
x\left(t_{k}^{+}\right) & =(1-\theta) x\left(t_{k}\right), \quad t=t_{k}, k \in N, \\
x\left(0^{+}\right) & =x_{0},
\end{aligned}
$$

where $a, b, \theta$ are positive constants, $t_{k+1}=t_{k}+\omega$. As a consequence of Lemma 3 , we have the following result: if $1-\theta-e^{-a \omega}>0$, then system (10) has a unique positive periodic solution $x^{*}(t)$, which is globally asymptotically stable. In fact, here $\int_{0}^{\omega} a d s+\ln (1-\theta)>0 \equiv 1-\theta-e^{-a \omega}>0$.

Lemma 5 (see [29]). Let $F: R_{+}^{n} \rightarrow R_{+}^{n}$ be continuous, $C^{1}$ in int $\left(R_{+}^{n}\right)$, and suppose $D F(0)$ exists with $\lim _{x \rightarrow 0^{+}} D F(z)=$ $\mathrm{DF}(0)$. In addition, assume

(a) $D F(x)>0$, if $x>0$;

(b) $D F(y)<D F(x)$, if $0<x<y$.

If $F(0)=0$, let $\lambda=\rho(D F(0))$. If $\lambda \leq 1$, then, for every $x \geq$ $0, F^{n}(x) \rightarrow 0$ as $n \rightarrow \infty$; if $\lambda>1$, then either $F^{n}(x) \rightarrow \infty$ as $n \rightarrow \infty$ for every $x>0$ or there exists a unique nonzero fixed point $q$ of $F$. In the latter case, $q>0$ and, for every $x>0$, $F^{n}(x) \rightarrow q$ as $n \rightarrow \infty$.

If $F(0) \neq 0$, then either $F^{n}(x) \rightarrow \infty$ as $n \rightarrow \infty$ for every $x \geq 0$ or there exists a unique fixed point $q$ of $F$. In the latter case, $q>0$ and, for every $x>0, F^{n}(x) \rightarrow q$ as $n \rightarrow \infty$.

Next, we analyze system (4). Integrating and solving the first two equations of system (4) between pulses, we have

$$
\begin{array}{r}
x_{i}(t)=\left[\frac{b_{i}}{a_{i}}+\left(\frac{1}{x_{i}\left(n \tau^{+}\right)}-\frac{b_{i}}{a_{i}}\right) e^{-a_{i}(t-n \tau)}\right]^{-1}, \\
n \tau<t \leq(n+1) \tau, i=1,2 .
\end{array}
$$

Similarly, considering the last two equations of system (4), we obtain the following stroboscopic map:

$$
\begin{aligned}
& x_{1}^{n+1}=\frac{\left(1-D_{1}\right) x_{1}^{n}}{h_{1}+c_{1} x_{1}^{n}}+\frac{d_{2} x_{2}^{n}}{h_{2} e^{a_{2} \tau_{0}}+l_{2} x_{2}^{n}}, \\
& x_{2}^{n+1}=\frac{\left(1-D_{2}\right) x_{2}^{n}}{h_{2}+c_{2} x_{2}^{n}}+\frac{d_{1} x_{1}^{n}}{h_{1} e^{a_{1} \tau_{0}}+l_{1} x_{1}^{n}} ;
\end{aligned}
$$


here $x_{i}^{n+1}=x_{i}\left[(n+1) \tau^{+}\right], c_{i}=\left(b_{i} / a_{i}\right)\left(1-e^{-a_{i} \tau}\right)>0, l_{i}=$ $\left(b_{i} / a_{i}\right)\left(1-e^{-a_{i}\left(\tau-\tau_{0}\right)}\right)>0,0<h_{i}=e^{-a_{i} \tau}<1$.

Remark 6. System (12) is a difference system, which means that densities of population in two patches have values at the previous pulse. We are, in other words, stroboscopically sampling at its pulsing period. The dynamical behavior of system (12), coupled with (11), determines the dynamical behavior of system (4). In the following sections, we will focus our attention on system (12) and investigate various aspects of its dynamical behavior. $R_{+}^{2}$ :

To write system (12) as a map, we define the map : $R_{+}^{2} \rightarrow$

$$
\begin{aligned}
& F_{1}\left(x_{1}, x_{2}\right)=\frac{\left(1-D_{1}\right) x_{1}}{h_{1}+c_{1} x_{1}}+\frac{d_{2} x_{2}}{h_{2} e^{a_{2} \tau_{0}}+l_{2} x_{2}}, \\
& F_{2}\left(x_{1}, x_{2}\right)=\frac{\left(1-D_{2}\right) x_{2}}{h_{2}+c_{2} x_{2}}+\frac{d_{1} x_{1}}{h_{1} e^{a_{1} \tau_{0}}+l_{1} x_{1}} .
\end{aligned}
$$

The set of all iterations of the map $F$ is equivalent to the set of all density sequences generated by system $(12) ; F(x)$ is the map evaluated at the point $x=\left(x_{1}, x_{2}\right) \in R_{+}^{2}$. Consequently, in system (12), $F^{n}$ describes the population densities in the time $n \tau$.

On the positivity of solutions of system (4) we have the following result.

Lemma 7. The solution $x\left(t, t_{0}, \phi\right)$ of system (4) with initial condition (5) is positive, that is, $x\left(t, t_{0}, \phi\right)>0$ on the interval of the existence.

The proof of Lemma 7 is simple; we hence omit it here.

\section{Permanence and Extinction}

In this section, we present conditions to ensure that system (12) is permanent and extinct which will imply the permanence and extinction of system (4). The permanence plays an important role in mathematical ecology since the criterion of permanence for ecological systems is a condition ensuring the long-term survival of all species. So, we firstly prove system (12) is permanent.

\section{Theorem 8. Suppose}

$$
1-D_{i}-h_{i}>0, \quad i=1,2,
$$

hold; then system (12) is permanent.

Proof. Let $x_{i}(t) \in P C^{\prime}$ be the solution of system (4) satisfying the initial conditions (5). From the first equation of system (12), we have

$$
\begin{aligned}
x_{1}^{n+1} & =\frac{1-D_{1}}{h_{1}\left(x_{1}^{n}\right)^{-1}+c_{1}}+\frac{d_{2}}{h_{2} e^{a_{2} \tau_{0}}\left(x_{2}^{n}\right)^{-1}+l_{2}} \\
& <\frac{1-D_{1}}{c_{1}}+\frac{d_{2}}{l_{2}} .
\end{aligned}
$$

Similarly, we have

$$
\begin{aligned}
x_{2}^{n+1} & =\frac{1-D_{2}}{h_{2}\left(x_{2}^{n}\right)^{-1}+c_{2}}+\frac{d_{1}}{h_{1} e^{a_{1} \tau_{0}}\left(x_{1}^{n}\right)^{-1}+l_{1}} \\
& <\frac{1-D_{2}}{c_{2}}+\frac{d_{1}}{l_{1}} .
\end{aligned}
$$

Hence, by (14) and (15) we know that system (12) has an ultimately upper bound.

Next, we prove that all the solutions of system (4) are ultimately below bounded. Since $d_{i} \geq 0 \quad(i=1,2)$, from the third equation of system (4), we have

$$
\begin{aligned}
x_{1}\left(n \tau^{+}\right) & =x_{1}\left(n \tau^{-}\right)+\Delta x_{1}(n \tau) \\
& =\left(1-D_{1}\right) x_{1}(n \tau)+d_{2} x_{2}\left(n \tau-\tau_{0}\right) \\
& \geq\left(1-D_{1}\right) x_{1}(n \tau) .
\end{aligned}
$$

Similarly,

$$
x_{2}\left(n \tau^{+}\right) \geq\left(1-D_{2}\right) x_{2}(n \tau) .
$$

Thus, system (4) becomes

$$
\begin{aligned}
\dot{x}_{1}(t) & =x_{1}(t)\left[a_{1}-b_{1} x_{1}(t)\right], \dot{x}_{2}(t) \\
& =x_{2}(t)\left[a_{2}-b_{2} x_{2}(t)\right], \quad t \neq n \tau, \\
x_{1}\left(n \tau^{+}\right) & \geq\left(1-D_{1}\right) x_{1}(n \tau), x_{2}\left(n \tau^{+}\right) \\
& \geq\left(1-D_{2}\right) x_{2}(n \tau), \quad t=n \tau .
\end{aligned}
$$

From (18), we find that there is no relation between $x_{1}(t)$ and $x_{2}(t)$. Therefore, we will discuss them, respectively;

$$
\begin{array}{ll}
\dot{x}_{1}(t)=x_{1}(t)\left[a_{1}-b_{1} x_{1}(t)\right], & t \neq n \tau, \\
x_{1}\left(n \tau^{+}\right) \geq\left(1-D_{1}\right) x_{1}(n \tau), & t=n \tau, \\
\dot{x}_{2}(t)=x_{2}(t)\left[a_{2}-b_{2} x_{2}(t)\right], & t \neq n \tau, \\
x_{2}\left(n \tau^{+}\right) \geq\left(1-D_{2}\right) x_{2}(n \tau), & t=n \tau .
\end{array}
$$

If $\left(\mathrm{H}_{1}\right)$ holds, from Remark 4, we can obtain that the auxiliary system

$$
\begin{array}{ll}
\dot{u}_{1}(t)=u_{1}(t)\left[a_{1}-b_{1} u_{1}(t)\right], & t \neq n \tau, \\
u_{1}\left(n \tau^{+}\right)=\left(1-D_{1}\right) u_{1}(n \tau), & t=n \tau,
\end{array}
$$

has a unique positive periodic solution $u_{1}^{*}(t)=x_{1}^{*}(t)$ which is globally asymptotically stable.

Let $u_{1}(t)$ be the solution of system (20) with initial value $u_{1}\left(0^{+}\right)=x_{1}\left(0^{+}\right)$. By Lemma 2 , we have

$$
x_{1}(t) \geq u_{1}(t), \quad \forall t \geq 0 .
$$

Hence, for any $\varepsilon>0$ sufficiently small, there exists a $T_{1}>0$ such that

$$
x_{1}(t) \geq x_{1}^{*}(t)-\varepsilon \triangleq m_{1}, \quad \text { for } t \geq T_{1} .
$$


Similarly, if $\left(\mathrm{H}_{1}\right)$ holds, for above $\varepsilon>0$, there exists a $T_{2}>0$ such that

$$
x_{2}(t) \geq x_{2}^{*}(t)-\varepsilon \triangleq m_{2}, \quad \text { for } t \geq T_{2} .
$$

Denote $m=\min \left\{m_{1}, m_{2}\right\}$ and $T=\max \left\{T_{1}, T_{2}\right\}$; then we have $x_{1}(t)>m$ and $x_{2}(t)>m, t \geq T$. Finally, we can determine that there exist constants $\alpha_{i}, \beta_{i}\left(0<\alpha_{i}<\beta_{i}\right)(i=1,2)$, such that $\alpha_{1} \leq \liminf _{n \rightarrow \infty} x_{1}^{n} \leq \limsup _{n \rightarrow \infty} x_{1}^{n} \leq \beta_{1}$, and $\alpha_{2} \leq \liminf _{n \rightarrow \infty} x_{2}^{n} \leq \limsup _{n \rightarrow \infty} x_{2}^{n} \leq \beta_{2}$. The proof of Theorem 8 is completed. extinct.

Next, we present condition to ensure that system (12) is

Theorem 9. System (12) is extinct if

$$
\begin{aligned}
& {\left[\frac{d_{1} d_{2}}{e^{\left(a_{1}+a_{2}\right) \tau_{0}}}-\left(1-D_{1}\right)\left(1-D_{2}\right)\right] e^{\left(a_{1}+a_{2}\right) \tau}} \\
& \quad+e^{a_{1} \tau}\left(1-D_{1}\right)+e^{a_{2} \tau}\left(1-D_{2}\right) \leq 1 .
\end{aligned}
$$

Proof. Let us consider the system (13). Obviously, $F\left(x_{1}, x_{2}\right)$ is continuous $C^{1}$ in int $\left(R_{+}^{2}\right)$, and $F(0,0)=0$. We obtain

$$
\begin{aligned}
& D F\left(x_{1}, x_{2}\right)=\left(\begin{array}{cc}
\frac{\left(1-D_{1}\right) h_{1}}{\left(h_{1}+c_{1} x_{1}\right)^{2}} & \frac{d_{2} h_{2} e^{a_{2} \tau_{0}}}{\left(h_{2} e^{a_{2} \tau_{0}}+l_{2} x_{2}\right)^{2}} \\
\frac{d_{1} h_{1} e^{a_{1} \tau_{0}}}{\left(h_{1} e^{a_{1} \tau_{0}}+l_{1} x_{1}\right)^{2}} & \frac{\left(1-D_{2}\right) h_{2}}{\left(h_{2}+c_{2} x_{2}\right)^{2}}
\end{array}\right), \\
& D F(0,0)=\left(\begin{array}{cc}
\frac{1-D_{1}}{h_{1}} & \frac{d_{2}}{h_{2} e^{a_{2} \tau_{0}}} \\
\frac{d_{1}}{h_{1} e^{a_{1} \tau_{0}}} & \frac{1-D_{2}}{h_{2}}
\end{array}\right) .
\end{aligned}
$$

Obviously, $\lim _{\left(x_{1}, x_{2}\right) \rightarrow(0,0)} D F\left(x_{1}, x_{2}\right)=D F(0,0)$; if $x>$ $0, D F(x)>0$; if $0<x<y, D F(y)<D F(x)$. We have the characteristic equation of $D F(0,0)$ :

$$
\begin{aligned}
\lambda^{2}- & \left(\frac{1-D_{1}}{h_{1}}+\frac{1-D_{2}}{h_{2}}\right) \lambda+\frac{\left(1-D_{1}\right)\left(1-D_{2}\right)}{h_{1} h_{2}} \\
- & \frac{d_{1} d_{2}}{h_{1} h_{2} e^{\left(a_{1}+a_{2}\right) \tau_{0}}}=0 .
\end{aligned}
$$

Let $\lambda=\rho(D F(0,0))$; then we have

$$
\begin{aligned}
& \lambda=\left(\frac{1-D_{1}}{h_{1}}+\frac{1-D_{2}}{h_{2}}\right. \\
&+\left(\left(\frac{1-D_{1}}{h_{1}}+\frac{1-D_{2}}{h_{2}}\right)^{2}-\frac{4\left(1-D_{1}\right)\left(1-D_{2}\right)}{h_{1} h_{2}}\right. \\
&\left.\left.\quad+\frac{4 d_{1} d_{2}}{h_{1} h_{2} e^{\left(a_{1}+a_{2}\right) \tau_{0}}}\right)^{1 / 2}\right) \times(2)^{-1}
\end{aligned}
$$

$$
\begin{aligned}
& =\left(\frac{1-D_{1}}{h_{1}}+\frac{1-D_{2}}{h_{2}}\right. \\
& \quad+\sqrt{\left.\left(\frac{1-D_{1}}{h_{1}}-\frac{1-D_{2}}{h_{2}}\right)^{2}+\frac{4 d_{1} d_{2}}{h_{1} h_{2} e^{\left(a_{1}+a_{2}\right) \tau_{0}}}\right) \times(2)^{-1}} .
\end{aligned}
$$

Assume $\lambda>1$; then by (27) we can obtain

$$
\frac{1-D_{1}}{h_{1}}+\frac{1-D_{2}}{h_{2}}+\frac{d_{1} d_{2}}{h_{1} h_{2} e^{\left(a_{1}+a_{2}\right) \tau_{0}}}-\frac{\left(1-D_{1}\right)\left(1-D_{2}\right)}{h_{1} h_{2}}>1 \text {; }
$$

that is,

$$
\begin{gathered}
{\left[\frac{d_{1} d_{2}}{e^{\left(a_{1}+a_{2}\right) \tau_{0}}}-\left(1-D_{1}\right)\left(1-D_{2}\right)\right] e^{\left(a_{1}+a_{2}\right) \tau}} \\
+e^{a_{1} \tau}\left(1-D_{1}\right)+e^{a_{2} \tau}\left(1-D_{2}\right)>1,
\end{gathered}
$$

which contradicts with (24). Therefore we have $\lambda \leq 1$. By Lemma 5, we can get $F^{n}\left(x_{1}, x_{2}\right) \rightarrow(0,0)$ as $n \rightarrow \infty$, which means that system (12) is extinct. This completes the proof.

\section{Existence and Uniqueness of Positive Periodic Solution}

In this part, we will prove the existence and uniqueness of the fixed points of system (12), which means that system (4) has a uniquely positive periodic solution.

Theorem 10. If $\left(H_{1}\right)$ holds, then there exists a unique positive fixed point $x^{*}=\left(x_{1}^{*}, x_{2}^{*}\right)$ of system (12).

Proof. Corresponding to (12), let us consider the following system:

$$
\begin{aligned}
& x_{1}=\frac{\left(1-D_{1}\right) x_{1}}{h_{1}+c_{1} x_{1}}+\frac{d_{2} x_{2}}{h_{2} e^{a_{2} \tau_{0}}+l_{2} x_{2}}, \\
& x_{2}=\frac{\left(1-D_{2}\right) x_{2}}{h_{2}+c_{2} x_{2}}+\frac{d_{1} x_{1}}{h_{1} e^{a_{1} \tau_{0}}+l_{1} x_{1}} .
\end{aligned}
$$

From (30), we have

$$
x_{1}-\frac{\left(1-D_{1}\right) x_{1}}{h_{1}+c_{1} x_{1}}>0, \quad x_{2}-\frac{\left(1-D_{2}\right) x_{2}}{h_{2}+c_{2} x_{2}}>0 ;
$$

hence

$$
\begin{aligned}
& x_{1}>\frac{1}{c_{1}}\left(1-D_{1}-h_{1}\right)=\xi, \\
& x_{2}>\frac{1}{c_{2}}\left(1-D_{2}-h_{2}\right)=\eta .
\end{aligned}
$$


From (30), we also obtain

$$
\begin{aligned}
& x_{2}=\frac{h_{2} e^{a_{2} \tau_{0}}\left[x_{1}-\left(\left(1-D_{1}\right) x_{1}\right) /\left(h_{1}+c_{1} x_{1}\right)\right]}{d_{2}-l_{2}\left[x_{1}-\left(\left(1-D_{1}\right) x_{1}\right) /\left(h_{1}+c_{1} x_{1}\right)\right]}, \\
& G\left(x_{1}, x_{2}\right)=\frac{d_{1} x_{1}}{h_{1} e^{a_{1} \tau_{0}}+l_{1} x_{1}}+\frac{\left(1-D_{2}\right) x_{2}}{h_{2}+c_{2} x_{2}}-x_{2} .
\end{aligned}
$$

Thus

$$
x_{2}(\xi)=0, \quad G(\xi)=\frac{d_{1} \xi}{h_{1} e^{a_{1} \tau_{0}}+l_{1} \xi}>0 .
$$

Let $x_{2} \rightarrow+\infty$; then $f\left(x_{1}\right)=x_{1}-\left(\left(1-D_{1}\right) x_{1}\right) /\left(h_{1}+c_{1} x_{1}\right) \rightarrow$ $d_{2} / l_{2}$. And $f\left(x_{1}\right)$ is an increasing function on the interval $[\xi,+\infty)$. Since $f(\xi)=0$, so there exists $\bar{x}_{1}>\xi$ such that $f\left(\bar{x}_{1}\right)=d_{2} / l_{2}$. We can easily find that $G\left(\bar{x}_{1}\right)<0$. By the zero theory of continuous function, there exists $\left(x_{1}^{*}, x_{2}^{*}\right)$ such that

$$
\begin{gathered}
\xi<x_{1}^{*}<\bar{x}_{1}, \quad G\left(x_{1}^{*}, x_{2}^{*}\right)=0, \\
x_{2}^{*}=\frac{h_{2} e^{a_{2} \tau_{0}}\left[x_{1}^{*}-\left(\left(1-D_{1}\right) x_{1}^{*}\right) /\left(h_{1}+c_{1} x_{1}^{*}\right)\right]}{d_{2}-l_{2}\left[x_{1}^{*}-\left(\left(1-D_{1}\right) x_{1}^{*}\right) /\left(h_{1}+c_{1} x_{1}^{*}\right)\right]} .
\end{gathered}
$$

Next, we will prove the uniqueness of the fixed point. It follows from (33) that we obtain

$$
\begin{aligned}
\frac{d x_{2}}{d x_{1}}= & \frac{d_{2} h_{2} e^{a_{2} \tau_{0}}\left[1-h_{1}\left(1-D_{1}\right) /\left(h_{1}+c_{1} x_{1}\right)^{2}\right]}{\left\{d_{2}-l_{2}\left[x_{1}-\left(\left(1-D_{1}\right) x_{1}\right) /\left(h_{1}+c_{1} x_{1}\right)\right]\right\}^{2}}, \\
\frac{d G}{d x_{1}}= & \frac{d_{1} h_{1} e^{a_{1} \tau_{0}}}{\left(h_{1} e^{a_{1} \tau_{0}}+l_{1} x_{1}\right)^{2}}+\left[\frac{h_{2}\left(1-D_{2}\right)}{\left(h_{2}+c_{2} x_{2}\right)^{2}}-1\right] \frac{d x_{2}}{d x_{1}}, \\
\frac{d G}{d x_{1}}=1 & \\
\times & \quad\left\{\left(d_{2}-l_{2}\left[x_{1}-\frac{\left(1-D_{1}\right) x_{1}}{h_{1}+c_{1} x_{1}}\right]\right\}^{2}\right. \\
& \left.\times\left(h_{1} e^{a_{1} \tau_{0}}+l_{1} x_{1}\right)^{2}\right)^{-1} \\
& *\left\{\begin{array}{l}
d_{1} h_{1} e^{a_{1} \tau_{0}}\left\{d_{2}-l_{2}\left[x_{1}-\frac{\left(1-D_{1}\right) x_{1}}{h_{1}+c_{1} x_{1}}\right]\right\}^{2} \\
+
\end{array}\right. \\
& \times\left[\begin{array}{l}
d_{2} h_{2} e^{a_{2} \tau_{0}}\left(h_{1} e^{a_{1} \tau_{0}}+l_{1} x_{1}\right)^{2} \\
\left.\left.1-\frac{h_{1}\left(1-D_{1}\right)}{\left(h_{1}+c_{1} x_{1}\right)^{2}}\right]\left[\frac{h_{2}\left(1-D_{2}\right)}{\left(h_{2}+c_{2} x_{2}\right)^{2}}-1\right]\right\} .
\end{array}\right.
\end{aligned}
$$

Let

$$
\begin{aligned}
\varphi(x)= & d_{1} h_{1} e^{a_{1} \tau_{0}}\left\{d_{2}-l_{2}\left[x_{1}-\frac{\left(1-D_{1}\right) x_{1}}{h_{1}+c_{1} x_{1}}\right]\right\}^{2} \\
& +d_{2} h_{2} e^{a_{2} \tau_{0}}\left(h_{1} e^{a_{1} \tau_{0}}+l_{1} x_{1}\right)^{2} \\
& \times\left[1-\frac{h_{1}\left(1-D_{1}\right)}{\left(h_{1}+c_{1} x_{1}\right)^{2}}\right]\left[\frac{h_{2}\left(1-D_{2}\right)}{\left(h_{2}+c_{2} x_{2}\right)^{2}}-1\right] ;
\end{aligned}
$$

then

$$
\begin{aligned}
\frac{d \varphi(x)}{d x_{1}} & \\
= & 2 d_{1} h_{1} e^{a_{1} \tau_{0}}\left\{d_{2}-l_{2}\left[x_{1}-\frac{\left(1-D_{1}\right) x_{1}}{h_{1}+c_{1} x_{1}}\right]\right\} \\
& \times\left\{-l_{2}\left[1-\frac{h_{1}\left(1-D_{1}\right)}{\left(h_{1}+c_{1} x_{1}\right)^{2}}\right]\right\} \\
+ & 2 l_{1} d_{2} h_{2} e^{a_{2} \tau_{0}}\left(h_{1} e^{a_{1} \tau_{0}}+l_{1} x_{1}\right) \\
& \times\left[1-\frac{h_{1}\left(1-D_{1}\right)}{\left(h_{1}+c_{1} x_{1}\right)^{2}}\right]\left[\frac{h_{2}\left(1-D_{2}\right)}{\left(h_{2}+c_{2} x_{2}\right)^{2}}-1\right] \\
+ & d_{2} h_{2} e^{a_{2} \tau_{0}}\left(h_{1} e^{a_{1} \tau_{0}}+l_{1} x_{1}\right)^{2} \\
\times & \frac{2 c_{1} h_{1}\left(1-D_{1}\right)}{\left(h_{1}+c_{1} x_{1}\right)^{3}}\left[\frac{h_{2}\left(1-D_{2}\right)}{\left(h_{2}+c_{2} x_{2}\right)^{2}}-1\right] \\
+ & d_{2} h_{2} e^{a_{2} \tau_{0}}\left(h_{1} e^{a_{1} \tau_{0}}+l_{1} x_{1}\right)^{2} \\
\times & {\left[1-\frac{h_{1}\left(1-D_{1}\right)}{\left(h_{1}+c_{1} x_{1}\right)^{2}}\right]\left[-\frac{2 c_{2} h_{2}\left(1-D_{2}\right)}{\left(h_{2}+c_{2} x_{2}\right)^{3}}\right] . }
\end{aligned}
$$

By (31), we have $\left(1-D_{1}\right) /\left(h_{1}+c_{1} x_{1}\right)<1$; since $h_{1} /\left(h_{1}+\right.$ $\left.c_{1} x_{1}\right)<1$, so $h_{1}\left(1-D_{1}\right) /\left(h_{1}+c_{1} x_{1}\right)^{2}<1$. Similarly, we have $h_{2}\left(1-D_{2}\right) /\left(h_{2}+c_{2} x_{2}\right)^{2}<1$. Therefore, we obtain $d \varphi(x) / d x<$ 0 , which implies that $\varphi(x)$ is a decreasing function on the interval $[\xi,+\infty)$.

Since

$$
\begin{aligned}
\varphi(\xi)= & d_{1} d_{2}^{2} h_{1} e^{a_{1} \tau_{0}}+d_{2} h_{2} e^{a_{2} \tau_{0}} \\
& \times\left[h_{1} e^{a_{1} \tau_{0}}+\frac{l_{1}}{c_{1}}\left(1-D_{1}-h_{1}\right)\right]^{2} \\
& \times \frac{1-D_{1}-h_{1}}{1-D_{1}} \frac{1-D_{2}-h_{2}}{h_{2}}>0 \\
\varphi\left(\bar{x}_{1}\right) & \\
= & d_{1} h_{1} e^{a_{1} \tau_{0}}\left\{d_{2}-l_{2}\left[\bar{x}_{1}-\frac{\left(1-D_{1}\right) \bar{x}_{1}}{h_{1}+c_{1} \bar{x}_{1}}\right]\right\}^{2} \\
& +d_{2} h_{2} e^{a_{2} \tau_{0}}\left(h_{1} e^{a_{1} \tau_{0}}+l_{1} \bar{x}_{1}\right)^{2} \\
& \times\left[1-\frac{h_{1}\left(1-D_{1}\right)}{\left(h_{1}+c_{1} \bar{x}_{1}\right)^{2}}\right]\left[\frac{h_{2}\left(1-D_{2}\right)}{\left(h_{2}+c_{2} x_{2}\left(\bar{x}_{1}\right)\right)^{2}}-1\right] \\
= & 0+d_{2} h_{2} e^{a_{2} \tau_{0}}\left(h_{1} e^{a_{1} \tau_{0}}+l_{1} \bar{x}_{1}\right)^{2} \\
& \times\left[1-\frac{h_{1}\left(1-D_{1}\right)}{\left(h_{1}+c_{1} \bar{x}_{1}\right)^{2}}\right](-1)<0,
\end{aligned}
$$

using the zero theory of continuous function, there exists a unique point $\xi_{1} \in\left(\xi, \bar{x}_{1}\right)$ such that $\varphi\left(\xi_{1}\right)=0$. Besides,

$$
\begin{gathered}
\varphi\left(x_{1}\right)>0, \quad \forall x_{1} \in\left(\xi, \xi_{1}\right), \\
\varphi\left(x_{1}\right)<0, \quad \forall x_{1} \in\left(\xi_{1},+\infty\right) ;
\end{gathered}
$$




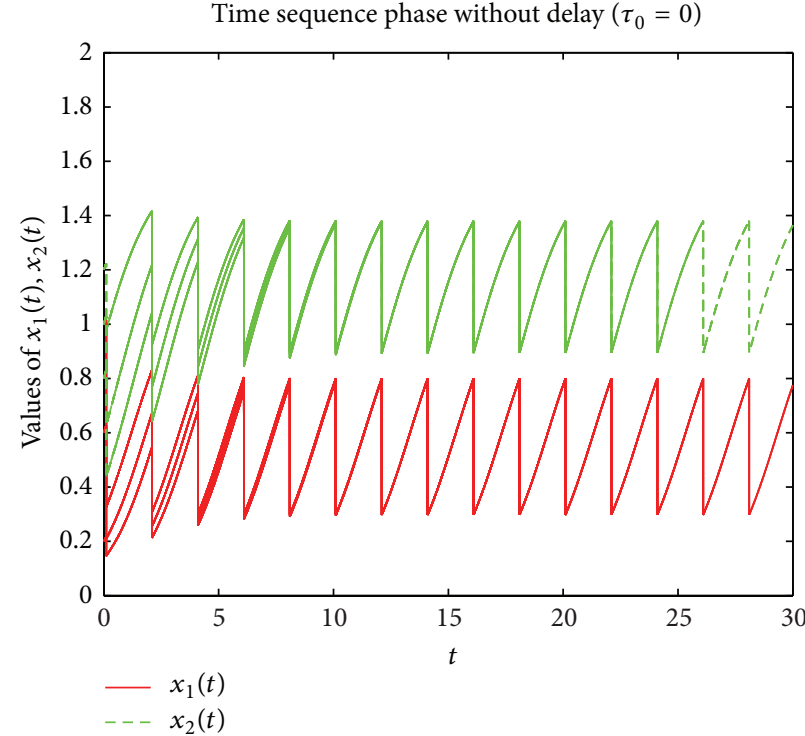

(a)

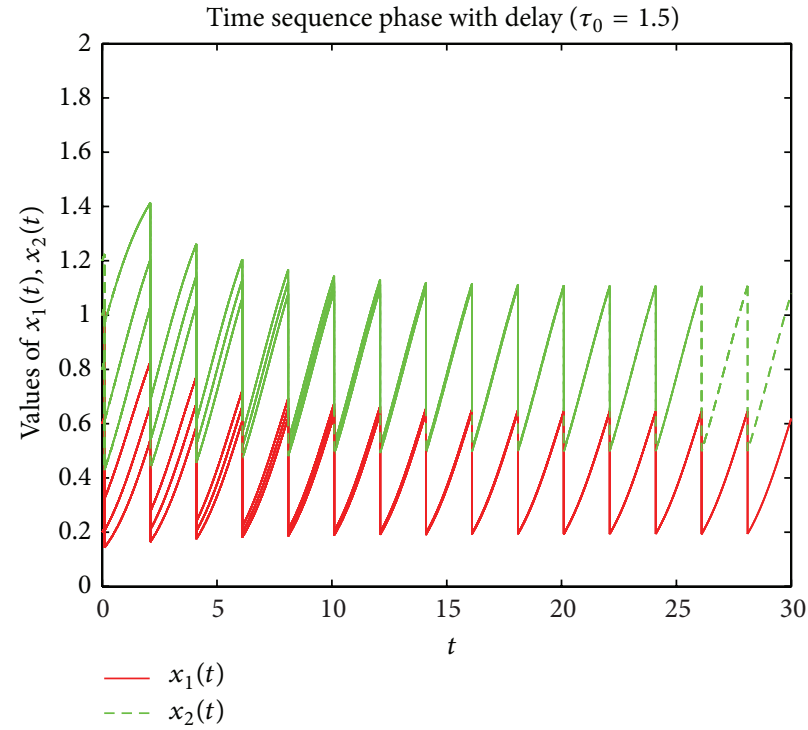

(c)

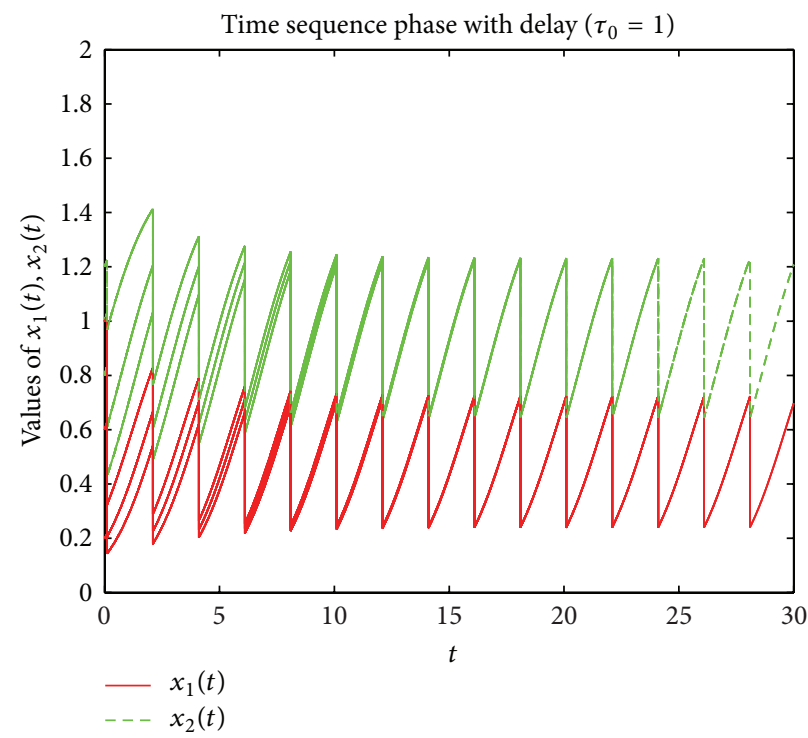

(b)

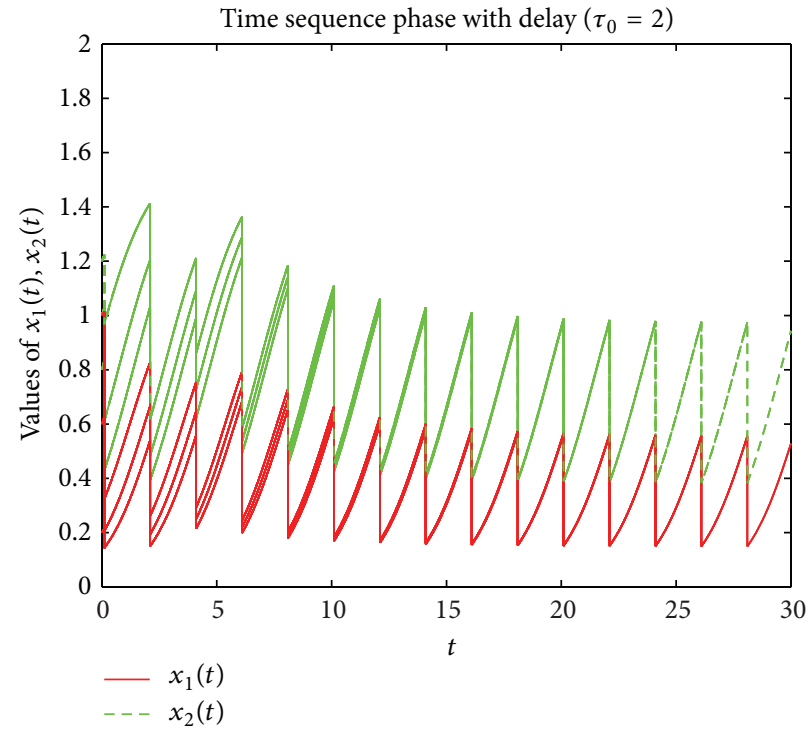

(d)

FIgURe 1: (a), (b), (c), and (d) Dynamical behavior of system (4). Here, we take three sets of initial values $(1,1.2),(0.6,0.8)$, and $(0.2,1)$.

thus

$$
\begin{gathered}
\frac{d G\left(x_{1}\right)}{d x_{1}}>0, \quad \forall x_{1} \in\left(\xi, \xi_{1}\right), \\
\frac{d G\left(x_{1}\right)}{d x_{1}}<0, \quad \forall x_{1} \in\left(\xi_{1},+\infty\right),
\end{gathered}
$$

which, together with $G(\xi)>0$, leads to $G\left(x_{1}\right)>0, \forall x_{1} \in$ $\left(\xi, \xi_{1}\right)$. By $G\left(\xi_{1}\right)>0, G\left(\bar{x}_{1}\right)<0$, we have that there exists a unique point $x_{1}^{*} \in\left(\xi_{1}, \bar{x}_{1}\right)$ such that $G\left(x_{1}^{*}, x_{2}^{*}\right)=0$. The proof is completed.

\section{Global Stability}

Now, we prove that the positive fixed points $\left(x_{1}^{*}, x_{2}^{*}\right)$ of system (30) are globally stable by using Lemma 5, which means that the positive periodic solution of system (4) is globally stable.

Theorem 11. If $\left(H_{1}\right)$ holds, then there exists a unique positive fixed point $x^{*}=\left(x_{1}^{*}, x_{2}^{*}\right)$ of the map $F$, and, for every $x=$ $\left(x_{1}, x_{2}\right)>0, F^{n}(x) \rightarrow x^{*}$ as $n \rightarrow \infty$.

Proof. For any small $\varepsilon_{1}>0, \varepsilon_{2}>0$, we make the change of variable

$$
x_{1}=u+\varepsilon_{1}, \quad x_{2}=v+\varepsilon_{2} .
$$




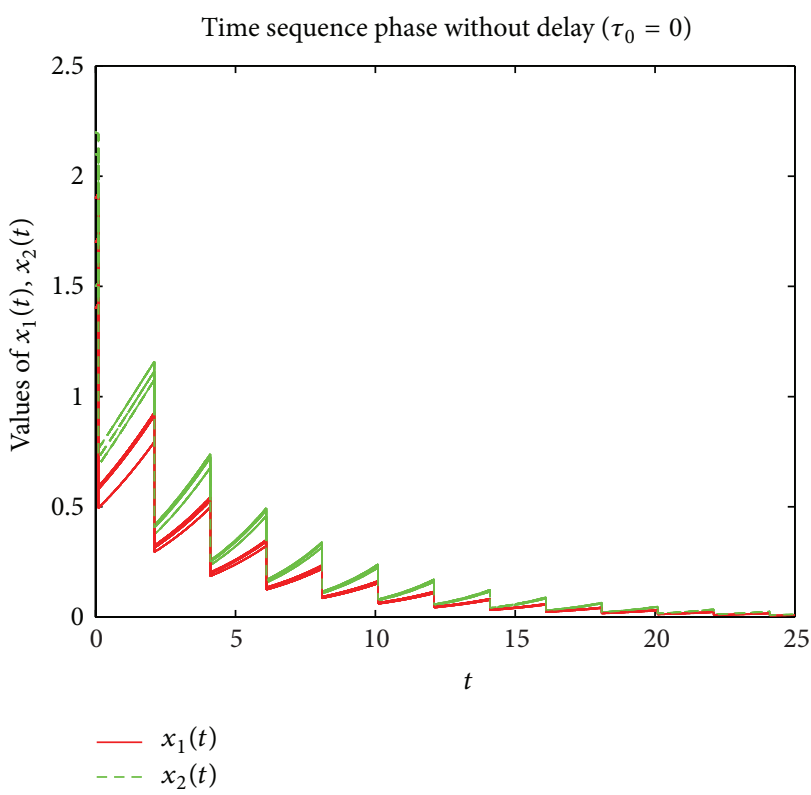

(a)

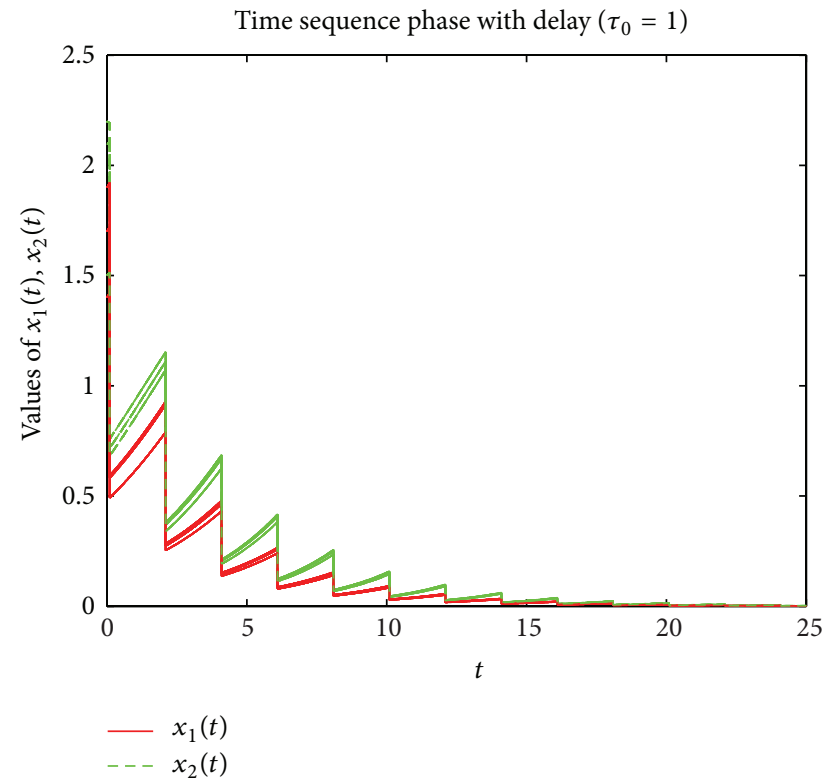

(b)

Figure 2: (a) and (b) Dynamical behavior of system (4). Here, we take three sets of initial values (1.7, 2.1), (1.9, 1.5), and (1.4, 2.2).

By (13), we get the map $F(u, v)=\left(F_{1}(u, v), F_{2}(u, v)\right)$; that is,

$$
\begin{aligned}
u= & \frac{\left(1-D_{1}\right)\left(u+\varepsilon_{1}\right)}{h_{1}+c_{1}\left(u+\varepsilon_{1}\right)}+\frac{d_{2}\left(v+\varepsilon_{2}\right)}{h_{2} e^{a_{2} \tau_{0}}+l_{2}\left(v+\varepsilon_{2}\right)} \\
& -\varepsilon_{1}=F_{1}(u, v), \\
v= & \frac{\left(1-D_{2}\right)\left(v+\varepsilon_{2}\right)}{h_{2}+c_{2}\left(v+\varepsilon_{2}\right)}+\frac{d_{1}\left(u+\varepsilon_{1}\right)}{h_{1} e^{a_{1} \tau_{0}}+l_{1}\left(u+\varepsilon_{1}\right)} \\
& -\varepsilon_{2}=F_{2}(u, v) .
\end{aligned}
$$

Now, we show that $F(u, v)$ satisfies the hypotheses of Lemma 5. It is easy to see that $F(u, v)$ is continuous, $C^{1}$ in int $\left(R_{+}^{2}\right)$, and $F(0,0) \neq 0$.

Since

$D F(u, v)$

$$
=\left(\begin{array}{cc}
\frac{\left(1-D_{1}\right) h_{1}}{\left[h_{1}+c_{1}\left(u+\varepsilon_{1}\right)\right]^{2}} & \frac{d_{2} h_{2} e^{a_{2} \tau_{0}}}{\left[h_{2} e^{a_{2} \tau_{0}}+l_{2}\left(v+\varepsilon_{2}\right)\right]^{2}} \\
\frac{d_{1} h_{1} e^{a_{1} \tau_{0}}}{\left[h_{1} e^{a_{1} \tau_{0}}+l_{1}\left(u+\varepsilon_{1}\right)\right]^{2}} & \frac{\left(1-D_{2}\right) h_{2}}{\left[h_{2}+c_{2}\left(v+\varepsilon_{2}\right)\right]^{2}}
\end{array}\right),
$$

DF $(0,0)$

$$
=\left(\begin{array}{cc}
\frac{\left(1-D_{1}\right) h_{1}}{\left(h_{1}+c_{1} \varepsilon_{1}\right)^{2}} & \frac{d_{2} h_{2} e^{a_{2} \tau_{0}}}{\left(h_{2} e^{a_{2} \tau_{0}}+l_{2} \varepsilon_{2}\right)^{2}} \\
\frac{d_{1} h_{1} e^{a_{1} \tau_{0}}}{\left(h_{1} e^{a_{1} \tau_{0}}+l_{1} \varepsilon_{1}\right)^{2}} & \frac{\left(1-D_{2}\right) h_{2}}{\left(h_{2}+c_{2} \varepsilon_{2}\right)^{2}}
\end{array}\right)
$$

so, $\lim _{(u, v) \rightarrow\left(0^{+}, 0^{+}\right)} D F(u, v)=D F(0,0)$. Obviously, if $(u, v)>$ 0 , then $D F(u, v)>0$; if $(0,0)<\left(u_{1}, v_{1}\right)<\left(u_{2}, v_{2}\right)$, then $D F\left(u_{1}, v_{1}\right)>D F\left(u_{2}, v_{2}\right)$. It satisfies all the conditions of Lemma 5; then, for every $u>0, v>0$, we have $F^{n}(u, v) \rightarrow$ $\left(x_{1}^{*}-\varepsilon_{1}, x_{2}^{*}-\varepsilon_{2}\right)$ as $n \rightarrow \infty$. Corresponding to $x-y$ coordinate, this means, for $x_{1}>\varepsilon_{1}, x_{2}>\varepsilon_{2}$, the system (30) tends to the unique fixed point.

It follows from the permanence of system (12) that we have $x_{1}^{n}>\varepsilon_{1}, x_{2}^{n}>\varepsilon_{2}$ for any initial value $\left(x_{1}\left(0^{+}\right), x_{2}\left(0^{+}\right)\right)>$ $(0,0)$.

From the above analysis, we can know that, for every $x_{1}\left(0^{+}\right)>0, x_{2}\left(0^{+}\right)>0$, the trajectory of system (12) will tend to $\left(x_{1}^{*}, x_{2}^{*}\right)$. The proof is completed.

\section{Numerical Simulation and Discussion}

In order to test the validity of our results, first, for (4) we use the parameters values (Val. 1) in Table 1. We can easily test that the assumptions in Theorems 8 and 10 hold, which means the populations $x(t)=\left(x_{1}(t), x_{2}(t)\right)$ in the two patches are permanent and have a unique periodic solution $x^{*}(t)=\left(x_{1}^{*}(t), x_{2}^{*}(t)\right)$ which is globally stable (see Figure 1(a)). Moreover, if, in Table 1, we consider the influence of time delay, then we can see that the permanence and stability for species $x$ unchanged. The details are given in Table 2. However, the longer the duration of the time delay, the lower the limit inferior and the limit superior of $x$ (see Figures 1(b), 1(c), and 1(d)). This implies that the case with dispersal delay is harmful to live for species $x$.

Next, we take the parameters values (Val. 2) in Table 1. We can easily test that assumption in Theorem $9\left[\left(d_{1} d_{2} / e^{\left(a_{1}+a_{2}\right) \tau_{0}}\right)-\left(1-D_{1}\right)\left(1-D_{2}\right)\right] e^{\left(a_{1}+a_{2}\right) \tau}+$ $e^{a_{1} \tau}\left(1-D_{1}\right)+e^{a_{2} \tau}\left(1-D_{2}\right)=0.5865<1$ holds, which 


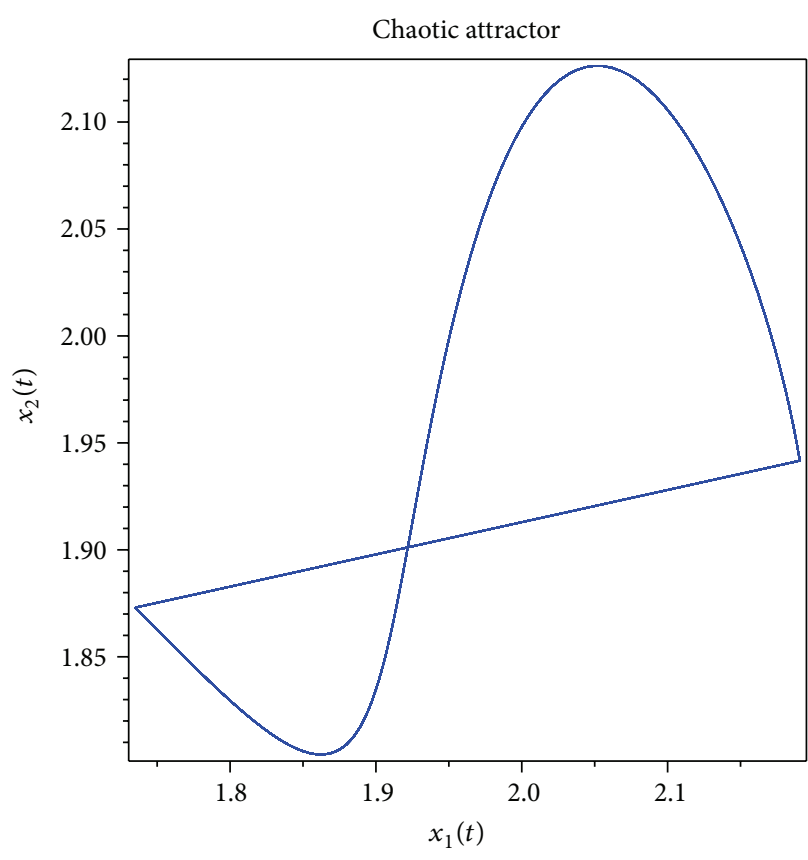

(a)

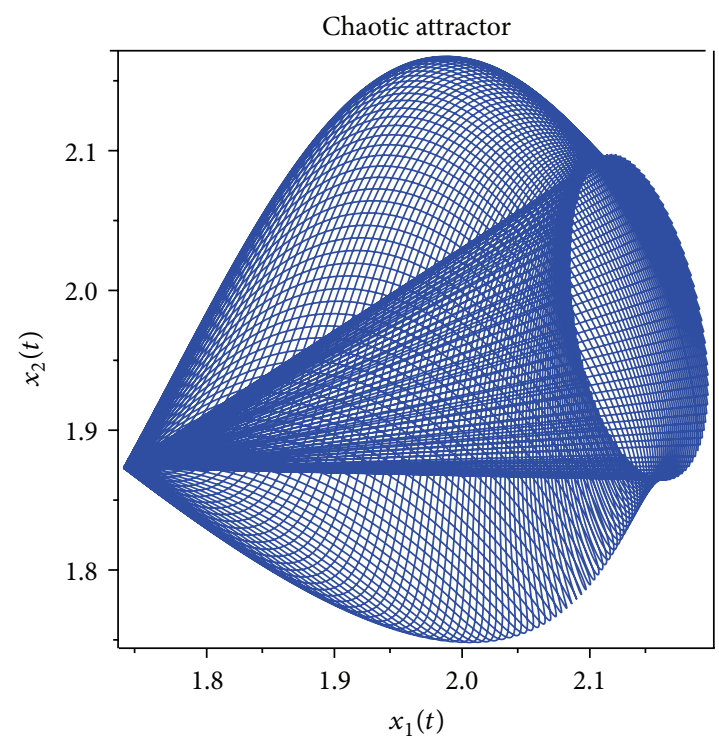

(c)

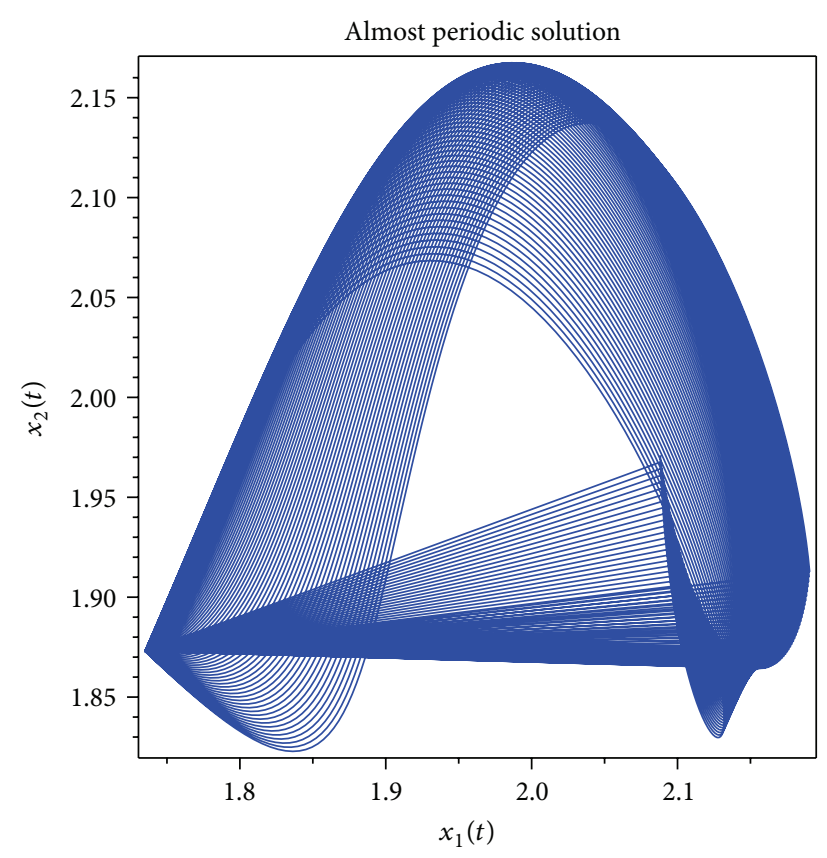

(b)

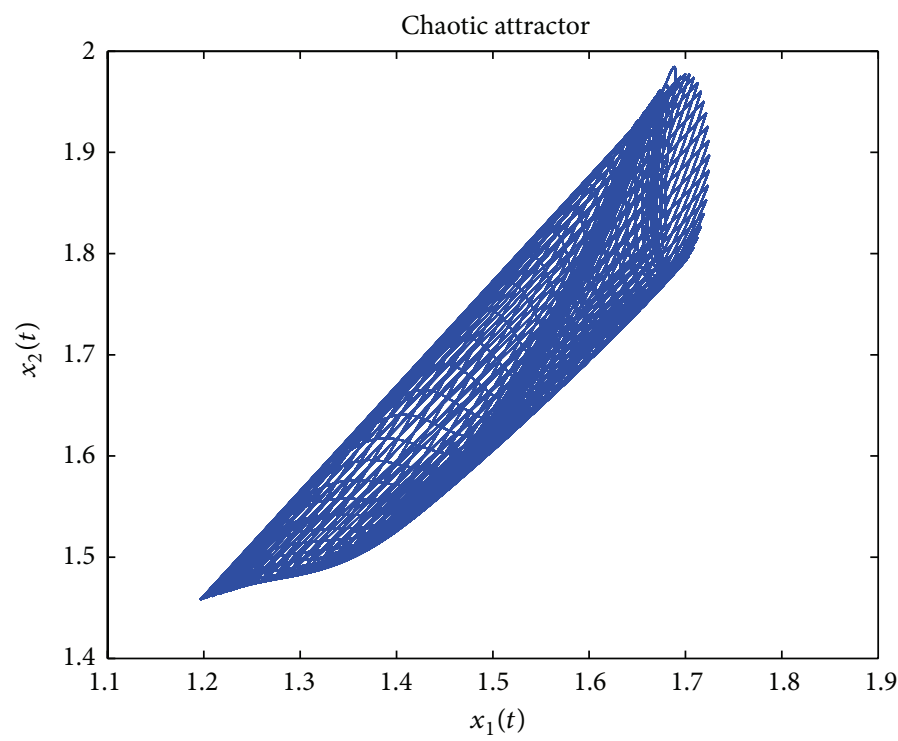

(d)

Figure 3: (a), (b), (c), and (d) Dynamical behavior of system (4). Here, we take the initial value $x_{0}=\left(x_{10}, x_{20}\right)=(1.735,1.873)$.

implies the populations $x(t)=\left(x_{1}(t), x_{2}(t)\right)$ in the two patches are extinct (see Figure 2). Comparing Figure 2(a) and Figure 2(b), we realize that system (4) with time delay accelerates the extinction comparing with no delay (see Figure 2). This is reasonable from a biological point of view. Without delay means less loss during dispersion, which implies that more members can arrive to other patches. Otherwise, populations go extinct due to much loss. The details are given in Table 3.

Furthermore, we take $a_{1}=1.1+0.04 \cos (\pi t), a_{2}=$ $0.8+0.01 \sin (\pi t), b_{1}=0.5+0.04 \sin (\pi t), b_{2}=0.4+$ $0.12 \cos (\pi t), d_{2}=0.55$ and keep other parameters unchanged from in Val. 1. Here we can see that the period of individual intrinsic growth rate and density dependence rate $T=$ 2 is equal to the period of migration $\tau$. In this case, we have let the period of the environment match the period of migration. If we take $\tau_{0}=0$, from simulation (see Figure 3(a)), we can see the populations $x(t)=\left(x_{1}(t), x_{2}(t)\right)$ are permanent and have a uniquely periodic solution which is globally stable. However, if we take the migration period $\tau=$ $2.009,2.022$, respectively, with other parameters unchanged, from numerical simulations (see Figures 3(b) and 3(c)), we can see that population dynamics change from almost periodic to chaotic. For Case 4 of Table 4, we consider the 


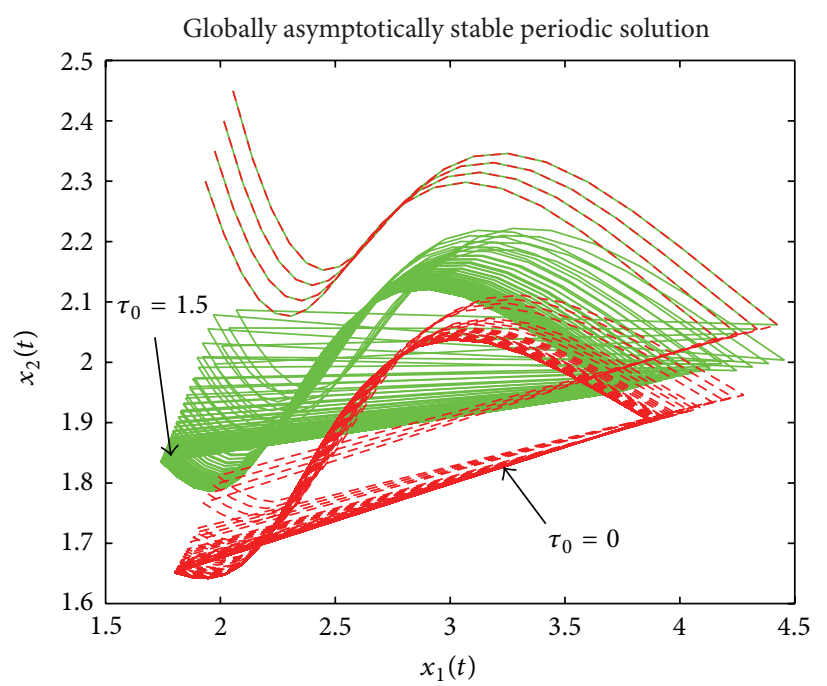

(a)

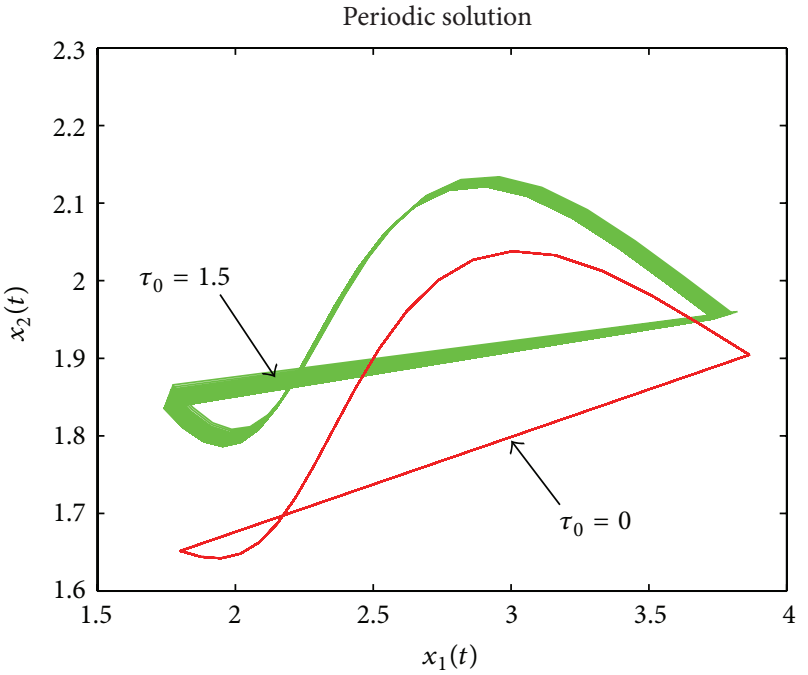

(b)

Figure 4: (a) and (b) Dynamical behavior of system (4). We take a series of initial points, such as $(1.935,2.3),(1.939,2.34)$, and (1.943,2.38).

TABLE 1: Parameter values used in the simulations of Model (4).

\begin{tabular}{llcc}
\hline Parameter & Interpretation & Val. 1 & Val. 2 \\
\hline$a_{1}$ & Intrinsic growth rate of populations in patch 1 & 0.9 & 0.3 \\
$a_{2}$ & Intrinsic growth rate of populations in patch 2 & 0.8 & 0.7 \\
$b_{1}$ & Density dependence rate of populations in patch 1 & 0.75 & 0.1 \\
$b_{2}$ & Density dependence rate of populations in patch 2 & 0.8 & 0.2 \\
$D_{1}$ & Successfully emigrant rate of populations from patch 1 & 0.7 & 0.9 \\
$D_{2}$ & Successfully emigrant rate of populations from patch 2 & 0.6 & 0.8 \\
$d_{1}$ & Successfully immigrant rate from patch 1 to patch 2 & 0.1 & 0.7 \\
$d_{2}$ & Successfully immigrant rate from patch 2 to patch 1 & 2 & 0.5 \\
$\tau$ & The period of dispersal between two pulse events & 0 & 2 \\
$\tau_{0}$ & Dispersal delay between two pulse events & & 1 \\
\hline
\end{tabular}

Val: value.

TABLE 2: Simulations of Model (4).

\begin{tabular}{lcccc}
\hline Case & $\tau_{0}$ & $x_{1}$ & $x_{2}$ & Figure \\
\hline 1 & 0 & Permanent & Permanent & Figure 1(a) \\
2 & 1 & Permanent & Permanent & Figure 1(b) \\
3 & 1.5 & Permanent & Permanent & Figure 1(c) \\
4 & 2 & Permanent & Permanent & Figure 1(d) \\
\hline
\end{tabular}

TABLE 3: Simulations of Model (4).

\begin{tabular}{lcccc}
\hline Case & $\tau_{0}$ & $x_{1}$ & $x_{2}$ & Figure \\
\hline 1 & 0 & Extinct & Extinct & Figure 2(a) \\
2 & 1 & Extinct & Extinct & Figure 2(b) \\
\hline
\end{tabular}

influence of time delay (see Figure 3(d)). Comparing Figures $3(\mathrm{c})$ and $3(\mathrm{~d})$, we realize that system (4) with time delay is more complicated than without. The details are given in Table 4 .
TABLE 4: Simulations of Model (4).

\begin{tabular}{lccccc}
\hline Case & $\tau$ & $\tau_{0}$ & $x_{1}$ & $x_{2}$ & Figure \\
\hline 1 & 2 & 0 & PP & PP & Figure 3(a) \\
2 & 2.009 & 0 & PAP & PAP & Figure 3(b) \\
3 & 2.022 & 0 & PC & PC & Figure 3(c) \\
4 & 2.05 & 1.1 & PC & PC & Figure 3(d) \\
\hline
\end{tabular}

PP: permanent and periodic, PAP: permanent and almost periodic, and PC: permanent and chaotic.

Lastly, if we take $a_{1}=0.9+0.04 \cos (\pi t)$ and keep other parameters unchanged with Figure 3 , by numerical simulations (see Figure 4), we find that all of the solutions of system (4) which through the initial points will converge to the positive periodic solution $\left(x_{1}^{*}, x_{2}^{*}\right)$. Therefore, we can guess that under the assumptions of Theorem 10 system (4) has a unique positive periodic solution which is globally asymptotically stable. In addition, the periodic solution with time delay is larger than without delay which indicates that 
the duration of the time delay is beneficial to live for species $x_{2}$ compared with species $x_{1}$.

\section{Conflict of Interests}

The authors declare that there is no conflict of interests regarding the publication of this paper.

\section{Acknowledgments}

This work was supported by the National Natural Science Foundation of China (nos. 10901130, 11361059, 10961022, and 11271312), the China Scholarship Council, the Natural Science Foundation of Xinjiang Province of China (2012211B07), and the Scientific Research Programmes of Colleges in Xinjiang (XJEDU2013I03).

\section{References}

[1] S. A. Levin, "Dispersion and population interactions," The American Naturalist, vol. 108, pp. 207-228, 1974.

[2] Z. Teng and Z. Lu, "The effect of dispersal on single-species nonautonomous dispersal models with delays," Journal of Mathematical Biology, vol. 42, no. 5, pp. 439-454, 2001.

[3] J. Cui, Y. Takeuchi, and Z. Lin, "Permanence and extinction for dispersal population systems," Journal of Mathematical Analysis and Applications, vol. 298, no. 1, pp. 73-93, 2004.

[4] J. Cui and L. Chen, "Permanence and extinction in logistic and Lotka-Volterra systems with diffusion," Journal of Mathematical Analysis and Applications, vol. 258, no. 2, pp. 512-535, 2001.

[5] R. Xu and Z. Ma, "The effect of dispersal on the permanence of a predator-prey system with time delay," Nonlinear Analysis. Real World Applications, vol. 9, no. 2, pp. 354-369, 2008.

[6] Y. Takeuchi, J. Cui, R. Miyazaki, and Y. Saito, "Permanence of delayed population model with dispersal loss," Mathematical Biosciences, vol. 201, no. 1-2, pp. 143-156, 2006.

[7] E. Beretta and Y. Takeuchi, "Global stability of single-species diffusion Volterra models with continuous time delays," Bulletin of Mathematical Biology, vol. 49, no. 4, pp. 431-448, 1987.

[8] E. Beretta and Y. Takeuchi, "Global asymptotic stability of Lotka-Volterra diffusion models with continuous time delay," SIAM Journal on Applied Mathematics, vol. 48, no. 3, pp. 627651, 1988.

[9] E. Beretta, P. Fergola, and C. Tenneriello, "Ultimate boundedness for nonautonomous diffusive Lotka-Volterra patches," Mathematical Biosciences, vol. 92, no. 1, pp. 29-53, 1988.

[10] H. I. Freedman, J. B. Shukla, and Y. Takeuchi, "Population diffusion in a two-patch environment," Mathematical Biosciences, vol. 95, no. 1, pp. 111-123, 1989.

[11] A. Hastings, "Dynamics of a single species in a spatially varying environment: the stabilizing role of high dispersal rates," Journal of Mathematical Biology, vol. 16, no. 1, pp. 49-55, 1982/83.

[12] W. Wang, L. Chen, and Z. Lu, "Global stability of a population dispersal in a two-patch environment," Dynamic Systems and Applications, vol. 6, no. 2, pp. 207-215, 1997.

[13] L. Dong, L. Chen, and P. Shi, "Periodic solutions for a twospecies nonautonomous competition system with diffusion and impulses," Chaos, Solitons \& Fractals, vol. 32, no. 5, pp. 19161926, 2007.
[14] J. Hui and L.-S. Chen, "A single species model with impulsive diffusion," Acta Mathematicae Applicatae Sinica, vol. 21, no. 1, pp. 43-48, 2005.

[15] L. Wang, Z. Liu, Jinghui, and L. Chen, "Impulsive diffusion in single species model," Chaos, Solitons \& Fractals, vol. 33, no. 4, pp. 1213-1219, 2007.

[16] G. Ballinger and X. Liu, "Permanence of population growth models with impulsive effects," Mathematical and Computer Modelling, vol. 26, no. 12, pp. 59-72, 1997.

[17] L. Zhang and Z. Teng, " $N$-species non-autonomous LotkaVolterra competitive systems with delays and impulsive perturbations," Nonlinear Analysis: Real World Applications, vol. 12, no. 6, pp. 3152-3169, 2011.

[18] J. Vandermeer, L. Stone, and B. Blasius, "Categories of chaos and fractal basin boundaries in forced predator-prey models," Chaos, Solitons \& Fractals, vol. 12, no. 2, pp. 265-276, 2001.

[19] L. Zhang, Z. Teng, D. L. DeAngelis, and S. Ruan, "Single species models with logistic growth and dissymmetric impulse dispersal," Mathematical Biosciences, vol. 241, no. 2, pp. 188-197, 2013.

[20] Z. Zhao, X. Zhang, and L. Chen, "The effect of pulsed harvesting policy on the inshore-offshore fishery model with the impulsive diffusion," Nonlinear Dynamics, vol. 63, no. 4, pp. 537-545, 2011.

[21] X.-P. Yan and W.-T. Li, "Hopf bifurcation and global periodic solutions in a delayed predator-prey system," Applied Mathematics and Computation, vol. 177, no. 1, pp. 427-445, 2006.

[22] L. Zhang and Z. Teng, "Permanence for a delayed periodic predator-prey model with prey dispersal in multi-patches and predator density-independent," Journal of Mathematical Analysis and Applications, vol. 338, no. 1, pp. 175-193, 2008.

[23] H. L. Smith, Monotone Dynamical Systems: An Introduction to the Theory of Competitive and Cooperative Systems, vol. 41 of Mathematical Surveys and Monographs, American Mathematical Society, Providence, RI, USA, 1995.

[24] X.-Q. Zhao, "Global attractivity in a class of nonmonotone reaction-diffusion equations with time delay," Canadian Applied Mathematics Quarterly, vol. 17, no. 1, pp. 271-281, 2009.

[25] Y. Takeuchi, W. Wang, and Y. Saito, "Global stability of population models with patch structure," Nonlinear Analysis: Real World Applications, vol. 7, no. 2, pp. 235-247, 2006.

[26] X. Meng and L. Chen, "Permanence and global stability in an impulsive Lotka-Volterra $n$-species competitive system with both discrete delays and continuous delays," International Journal of Biomathematics, vol. 1, no. 2, pp. 179-196, 2008.

[27] H. I. Freedman, B. Rai, and P. Waltman, "Mathematical models of population interactions with dispersal. II. Differential survival in a change of habitat," Journal of Mathematical Analysis and Applications, vol. 115, no. 1, pp. 140-154, 1986.

[28] R. H. MacArthur and E. O. Wilson, The Theory of Island Biogeography, Princeton University Press, 1967.

[29] H. L. Smith, "Cooperative systems of differential equations with concave nonlinearities," Nonlinear Analysis: Theory, Methods \& Applications, vol. 10, no. 10, pp. 1037-1052, 1986.

[30] V. Lakshmikantham, D. D. Baĭnov, and P. S. Simeonov, Theory of Impulsive Differential Equations, vol. 6 of Series in Modern Applied Mathematics, World Scientific, Teaneck, NJ, USA, 1989.

[31] D. Bainov and P. Simeonov, Impulsive Differential Equations: Periodic Solutions and Applications, Longman, London, UK, 2003.

[32] F. Zhang, S. Gao, and Y. Zhang, "Effects of pulse culling on population growth of migratory birds and economical birds," Nonlinear Dynamics, vol. 67, no. 1, pp. 767-779, 2012. 


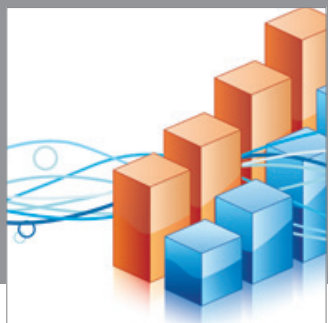

Advances in

Operations Research

mansans

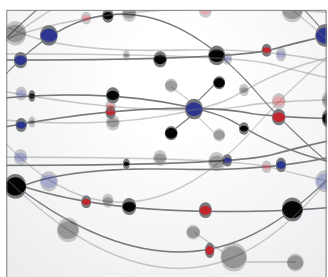

The Scientific World Journal
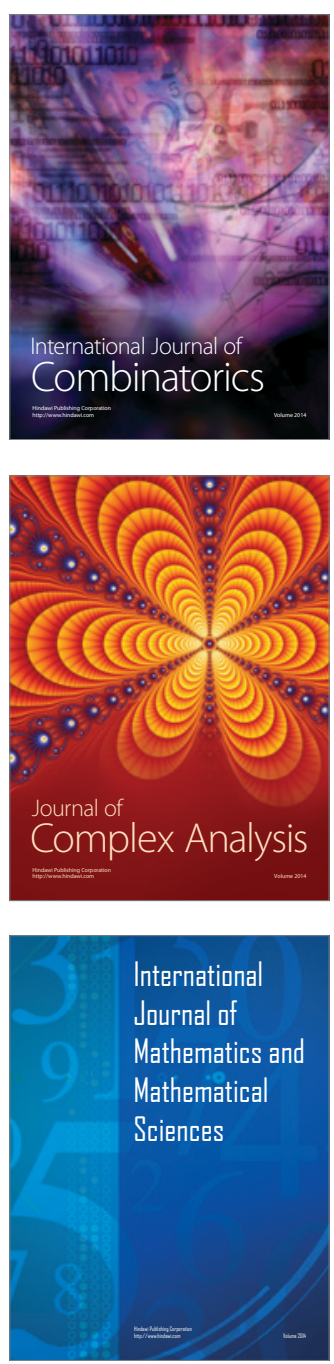
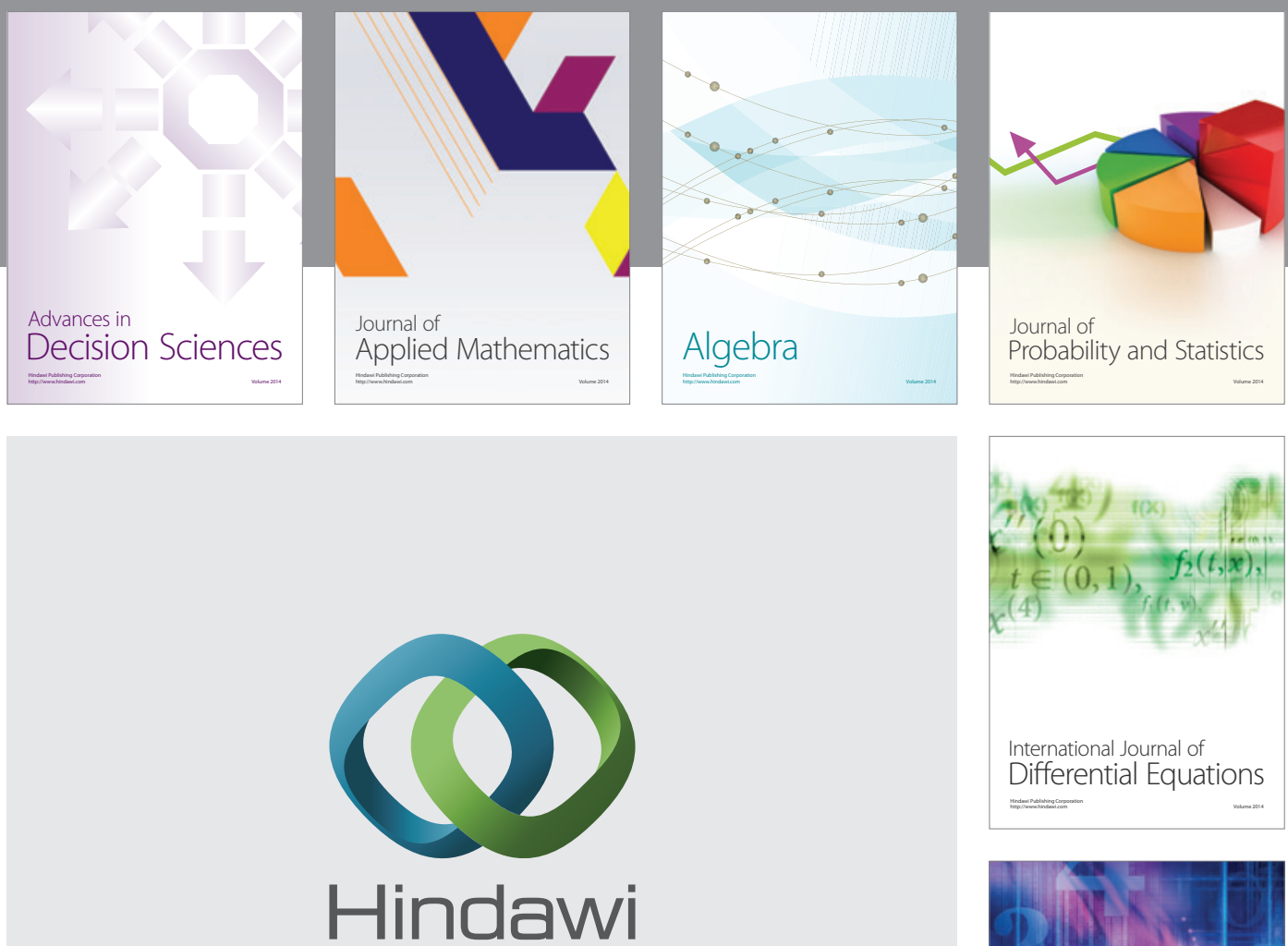

Submit your manuscripts at http://www.hindawi.com
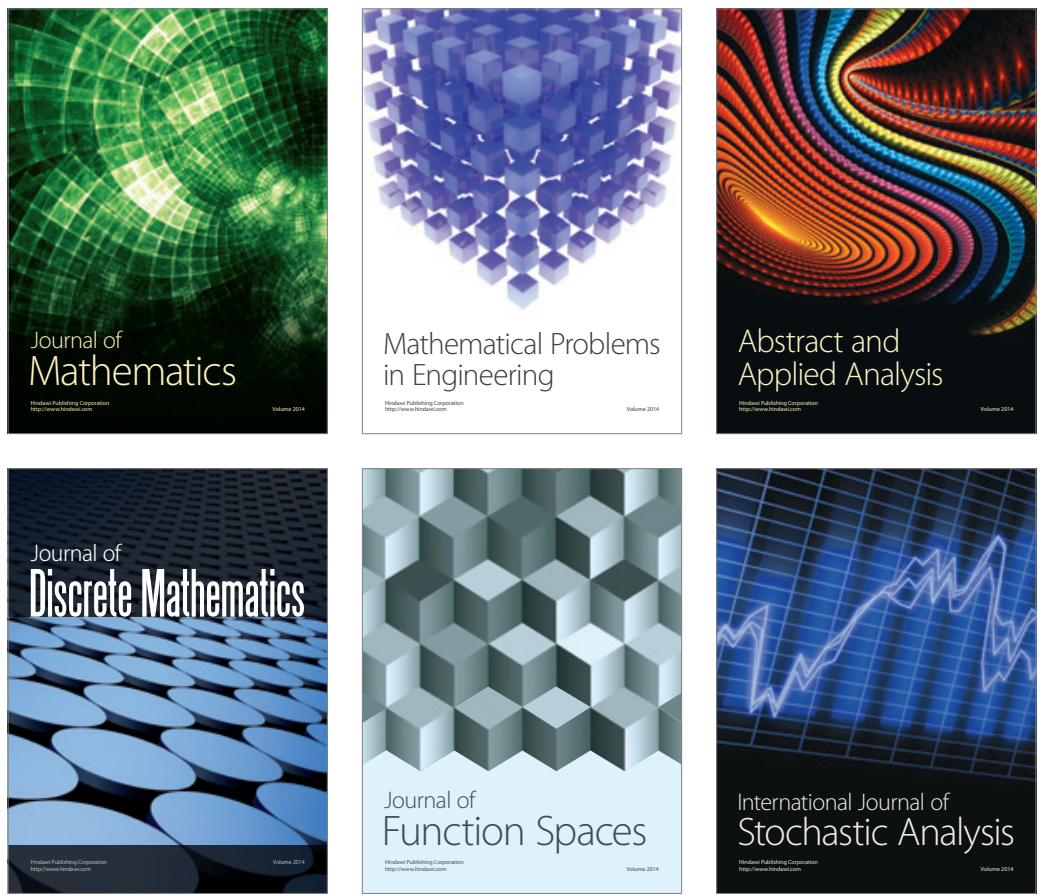

Journal of

Function Spaces

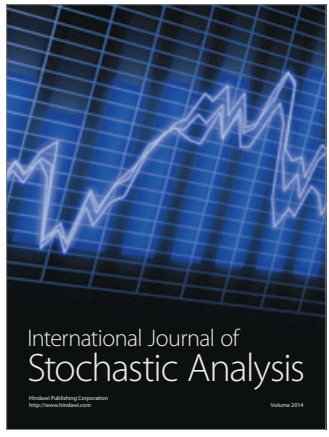

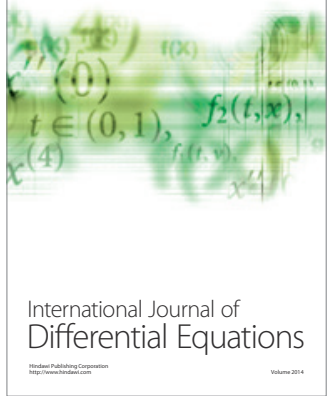
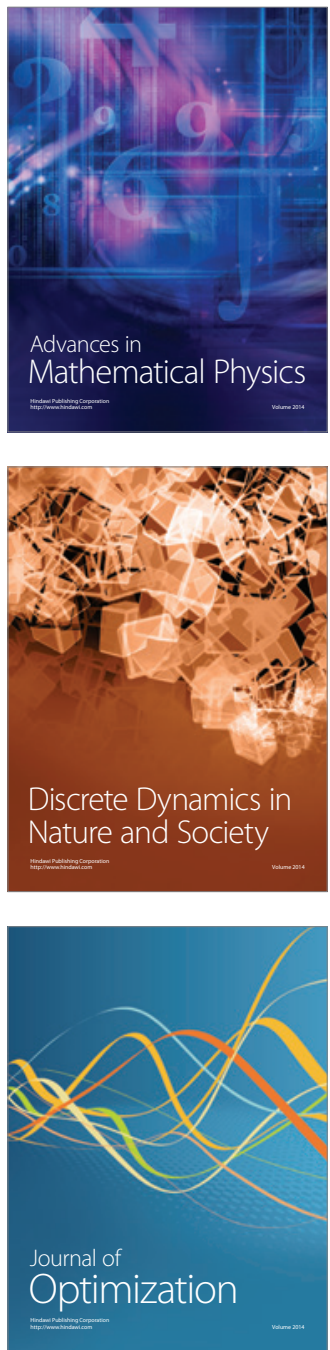